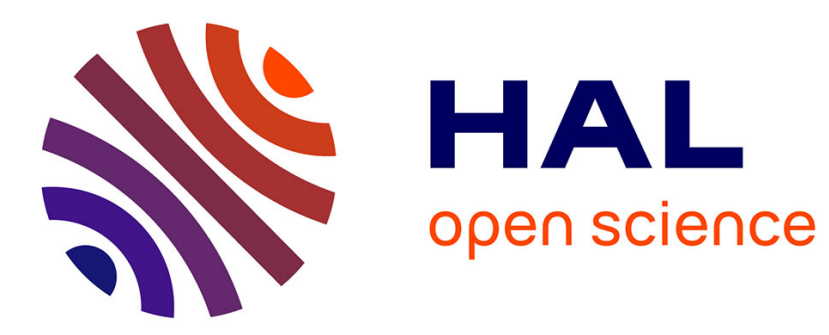

\title{
Black-Box Optimization Benchmarking the IPOP-CMA-ES on the Noiseless Testbed
}

\author{
Raymond Ros
}

\section{To cite this version:}

Raymond Ros. Black-Box Optimization Benchmarking the IPOP-CMA-ES on the Noiseless Testbed. Genetic and Evolutionary Computation Conference 2010, Jul 2010, Portland, OR, United States. inria-00473777

\section{HAL Id: inria-00473777 \\ https://hal.inria.fr/inria-00473777}

Submitted on 16 Apr 2010

HAL is a multi-disciplinary open access archive for the deposit and dissemination of scientific research documents, whether they are published or not. The documents may come from teaching and research institutions in France or abroad, or from public or private research centers.
L'archive ouverte pluridisciplinaire HAL, est destinée au dépôt et à la diffusion de documents scientifiques de niveau recherche, publiés ou non, émanant des établissements d'enseignement et de recherche français ou étrangers, des laboratoires publics ou privés. 


\title{
Black-Box Optimization Benchmarking the IPOP-CMA-ES on the Noiseless Testbed
}

\author{
Comparison to the BIPOP-CMA-ES
}

\author{
Raymond Ros \\ TAO Team-Project - INRIA Saclay \\ LRI, Bat 490, Univ. Paris-Sud \\ F-91405 Orsay Cedex, France \\ raymond.ros@inria.fr
}

\begin{abstract}
We benchmark the Covariance Matrix Adaptation-Evolution Strategy (CMA-ES) algorithm with an Increasing $\mathrm{POPu}$ lation size (IPOP) restart policy on the $\mathrm{BBOB}$ noiseless testbed. The IPOP-CMA-ES is compared to the BIPOPCMA-ES and is shown to perform at best two times faster on multi-modal functions $f_{15}$ to $f_{19}$ whereas it does not solve weakly structured functions $f_{22}, f_{23}$ and $f_{24}$.
\end{abstract}

\section{Categories and Subject Descriptors}

G.1.6 [Numerical Analysis]: Optimization-global optimization, unconstrained optimization; F.2.1 [Analysis of Algorithms and Problem Complexity]: Numerical Algorithms and Problems

\section{General Terms}

Algorithms

\section{Keywords}

Benchmarking, Black-box optimization, Evolution strategy

\section{ALGORITHM PRESENTATION}

The algorithm Covariance Matrix Adaptation-Evolution Strategy (CMA-ES) [9] is a stochastic search method based on a population. We choose to apply the $\left(\mu / \mu_{w}, \lambda\right)$-CMAES $[3,7,8]$ in this paper. The Increasing POPulation-size (IPOP) restart policy was proposed for the CMA-ES in [1]. The resulting IPOP-CMA-ES algorithm uses a population doubling in size at each restarts.

We compare the performances of the IPOP-CMA-ES to those of the BIPOP-CMA-ES [4] which was proposed to the BBOB 2009 workshop. The BIPOP-CMA-ES distributes the allocated budget - number of function evaluations - between a doubling population size and a small population size

Permission to make digital or hard copies of all or part of this work for personal or classroom use is granted without fee provided that copies are not made or distributed for profit or commercial advantage and that copies bear this notice and the full citation on the first page. To copy otherwise, to republish, to post on servers or to redistribute to lists, requires prior specific permission and/or a fee.

GECCO'10, July 7-11, 2010, Portland, Oregon, USA

Copyright 2010 ACM 978-1-4503-0073-5/10/07 ...\$10.00. policy. The BIPOP-CMA-ES showed good performances on the function testbeds of the BBOB 2009 workshop [4].

The implementation of the IPOP-CMA-ES that we benchmark is the version 3.40beta of the Matlab code available

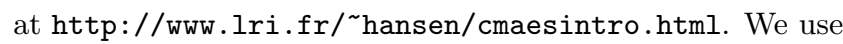
the parameter settings described in [4] for the BIPOP-CMAES. Therefore, the only difference between BIPOP-CMA-ES and IPOP-CMA-ES is that all the allocated budget is assigned to the doubling population size restart policy.

No additional parameter tuning has been done, the crafting effort [5] of IPOP-CMA-ES computes to $\mathrm{CrE}=0$, which was also the case for the BIPOP-CMA-ES.

\section{CPU TIMING EXPERIMENT}

The complete algorithms were run on $f_{8}$ for at least 30 seconds. Results for the IPOP-CMA-ES are $1.8 ; 1.5 ; 1.3$; $1.1 ; 1.1 ; 1.5$ and $3.4 \times 10^{-4}$ seconds per function evaluation for dimension $2 ; 3 ; 5 ; 10 ; 20 ; 40$ and 80 . These figures were obtained on a Intel Core 26700 processor $(2.66 \mathrm{GHz})$ with Linux 2.6.28-18 and Matlab R2008a.

\section{RESULTS}

The data for BIPOP-CMA-ES were obtained using the BBOB 2009 experimental set-up which differ from that of BBOB 2010 only in the number of test function instances considered (respectively 1 to 5 for BBOB 2009 and 1 to 15 for BBOB 2010) and the number of repetitions on each of these function instances (resp. 3 for BBOB 2009 and 1 for BBOB 2010).

Results from experiments according to [5] on the benchmark functions given in [2,6] are presented in Figures 1, 2, 3 and 4 and in Tables 1 and 2 . The expected running time (ERT), used in the figures and tables, depends on a given target function value, $f_{\mathrm{t}}=f_{\mathrm{opt}}+\Delta f$, and is computed over all relevant trials as the number of function evaluations executed during each trial while the best function value did not reach $f_{\mathrm{t}}$, summed over all trials and divided by the number of trials that actually reached $f_{\mathrm{t}}[5,10]$. Statistical significance is tested with the rank-sum test for a given target $\Delta f_{\mathrm{t}}\left(10^{-8}\right.$ in Figure 1) using, for each trial, either the number of needed function evaluations to reach $\Delta f_{\mathrm{t}}$ (inverted and multiplied by -1 ), or, if the target was not reached, the best $\Delta f$-value achieved, measured only up to the smallest number of overall function evaluations for any unsuccessful trial under consideration.

Figure 3 shows that the proportion of functions solved by 
BIPOP-CMA-ES is larger than IPOP-CMA-ES. The most prominent differences between the performances of the two algorithms are in the group of the multi-modal functions $\left(f_{15}\right.$ to $\left.f_{19}\right)$ and that of the weakly structured multi-modal functions $\left(f_{20}\right.$ to $\left.f_{24}\right)$.

The IPOP-CMA-ES is shown to perform faster on functions $f_{7}, f_{13}, f_{15}, f_{16}, f_{17}, f_{18}, f_{19}$ by a factor of around two at most when the dimension of the search space is larger than 10. The IPOP-CMA-ES solves function $f_{19}$ but is slower than the BIPOP-CMA-ES in dimension smaller than 5. The IPOP-CMA-ES does not solve functions $f_{22} f_{23}$ and $f_{24}$ when the dimension is larger than 10 , whereas the BIPOP-CMA-ES does. The fact that BIPOP-CMA-ES can solve $f_{23}$ and $f_{24}$ can be attributed to the small population size management of BIPOP-CMA-ES. Finally, neither the IPOP-CMA-ES nor the BIPOP-CMA-ES solve functions $f_{3}$ when the dimension of the search space is larger than 10 , $f_{4}$ when the dimension is larger than 3 and $f_{20}$ when the dimension is larger than 40 .

\section{REFERENCES}

[1] A. Auger and N. Hansen. A restart CMA evolution strategy with increasing population size. In Proceedings of the IEEE Congress on Evolutionary Computation (CEC 2005), pages 1769-1776. IEEE Press, 2005.

[2] S. Finck, N. Hansen, R. Ros, and A. Auger. Real-parameter black-box optimization benchmarking 2009: Presentation of the noiseless functions. Technical Report 2009/20, Research Center PPE, 2009. Updated February 2010.

[3] N. Hansen. The CMA evolution strategy: a comparing review. In J. Lozano, P. Larranaga, I. Inza, and E. Bengoetxea, editors, Towards a new evolutionary computation. Advances on estimation of distribution algorithms, pages 75-102. Springer, 2006.

[4] N. Hansen. Benchmarking a BI-population CMA-ES on the BBOB-2009 function testbed. In F. Rothlauf, editor, GECCO (Companion), pages 2389-2396. ACM, 2009.

[5] N. Hansen, A. Auger, S. Finck, and R. Ros. Real-parameter black-box optimization benchmarking 2010: Experimental setup. Technical Report RR-7215, INRIA, 2010.

[6] N. Hansen, S. Finck, R. Ros, and A. Auger. Real-parameter black-box optimization benchmarking 2009: Noiseless functions definitions. Technical Report RR-6829, INRIA, 2009. Updated February 2010.

[7] N. Hansen and S. Kern. Evaluating the CMA evolution strategy on multimodal test functions. In X. Yao et al., editors, Parallel Problem Solving from Nature - PPSN VIII, LNCS 3242, pages 282-291. Springer, 2004.

[8] N. Hansen, A. Niederberger, L. Guzzella, and P. Koumoutsakos. A method for handling uncertainty in evolutionary optimization with an application to feedback control of combustion. IEEE Transactions on Evolutionary Computation, 13(1):180-197, 2009.

[9] N. Hansen and A. Ostermeier. Completely derandomized self-adaptation in evolution strategies. Evolutionary Computation, 9(2):159-195, 2001.

[10] K. Price. Differential evolution vs. the functions of the second ICEO. In Proceedings of the IEEE International Congress on Evolutionary Computation, pages 153-157, 1997. 

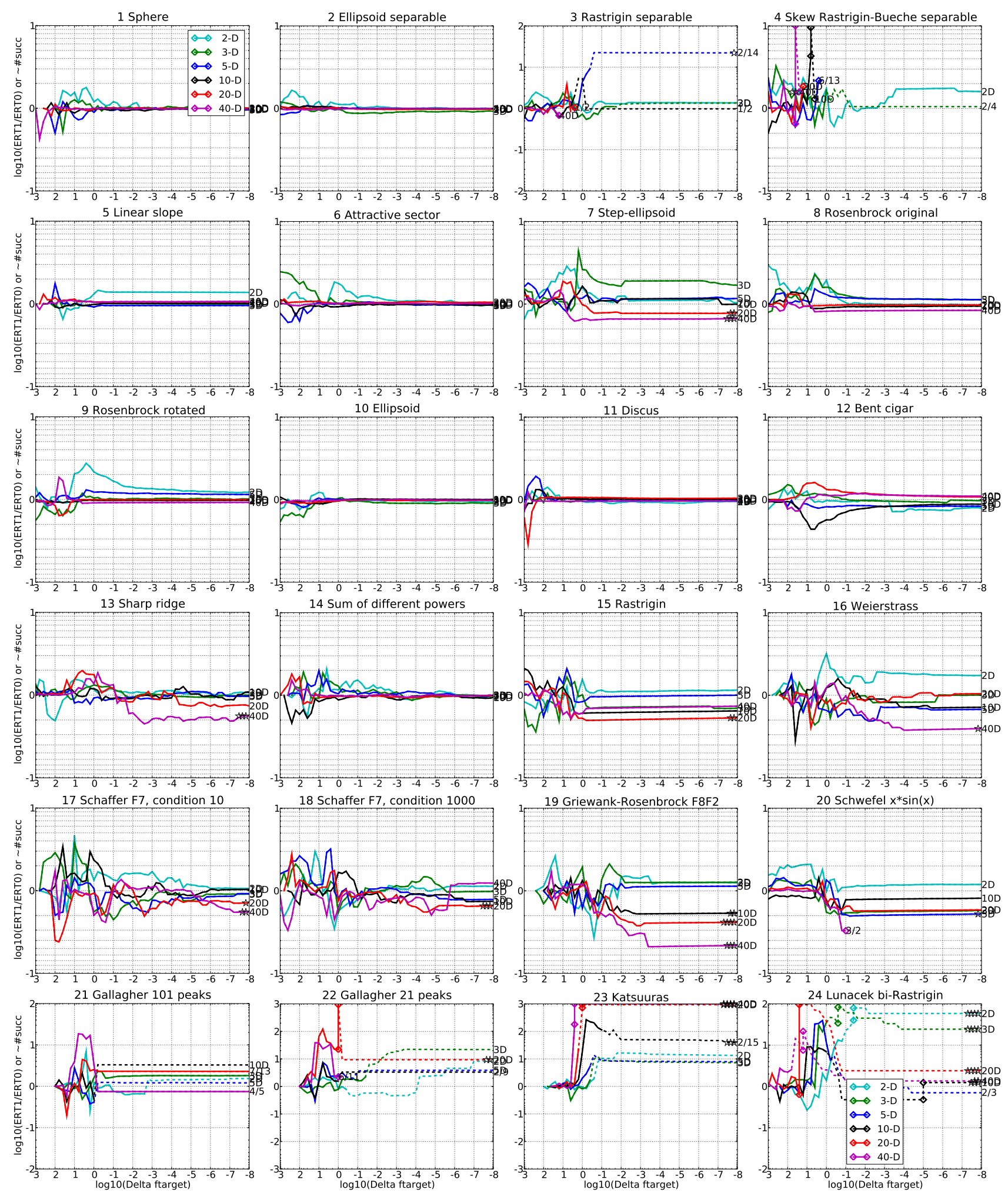

Figure 1: ERT ratio of IPOP-CMA divided by BIPOP-CMA versus $\log _{10}(\Delta f)$ for $f_{1}-f_{24}$ in $2,3, \mathbf{5}, \mathbf{1 0}, 20$, 40-D. Ratios $<10^{\circ}$ indicate an advantage of IPOP-CMA, smaller values are always better. The line gets dashed when for any algorithm the ERT exceeds thrice the median of the trial-wise overall number of $f$ evaluations for the same algorithm on this function. Symbols indicate the best achieved $\Delta f$-value of one algorithm (ERT gets undefined to the right). The dashed line continues as the fraction of successful trials of the other algorithm, where 0 means $0 \%$ and the y-axis limits mean $100 \%$, values below zero for IPOP-CMA. The line ends when no algorithm reaches $\Delta f$ anymore. The number of successful trials is given, only if it was in $\{1 \ldots 9\}$ for IPOP-CMA (1st number) and non-zero for BIPOP-CMA (2nd number). Results are significant with $p=0.05$ for one star and $p=10^{-\# \star}$ otherwise, with Bonferroni correction within each figure. 

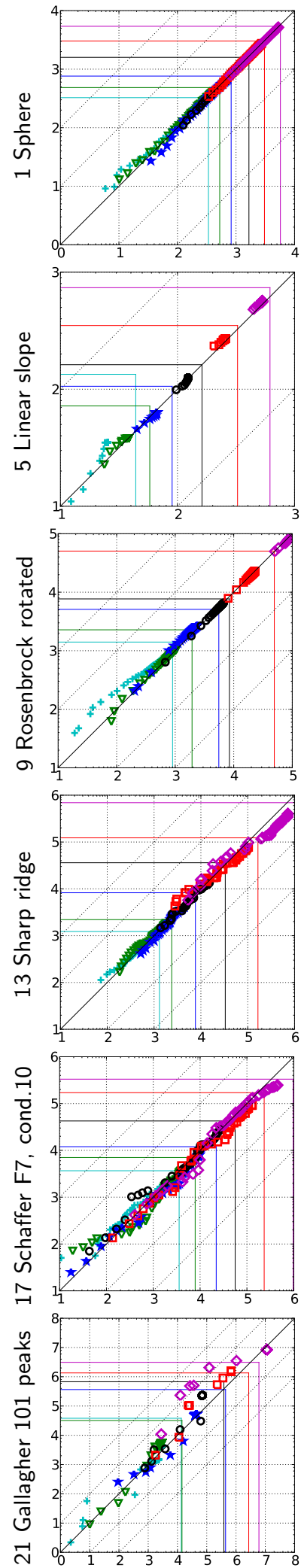
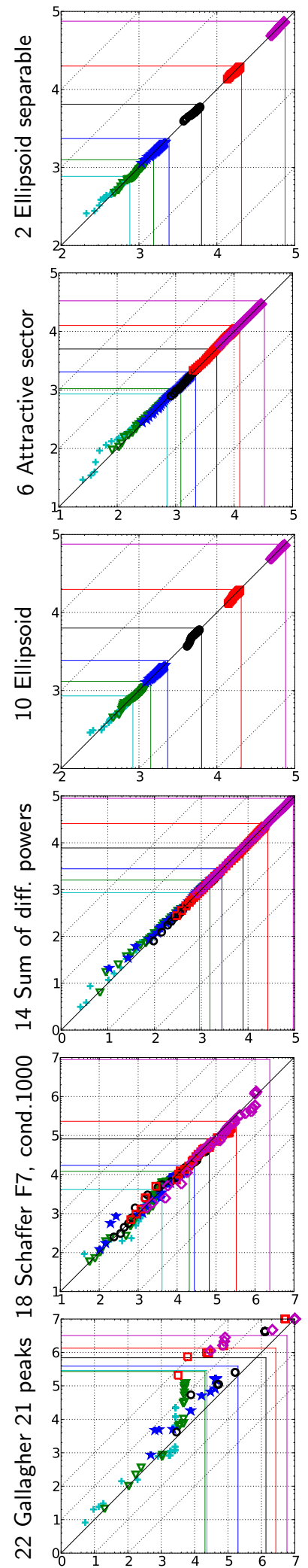
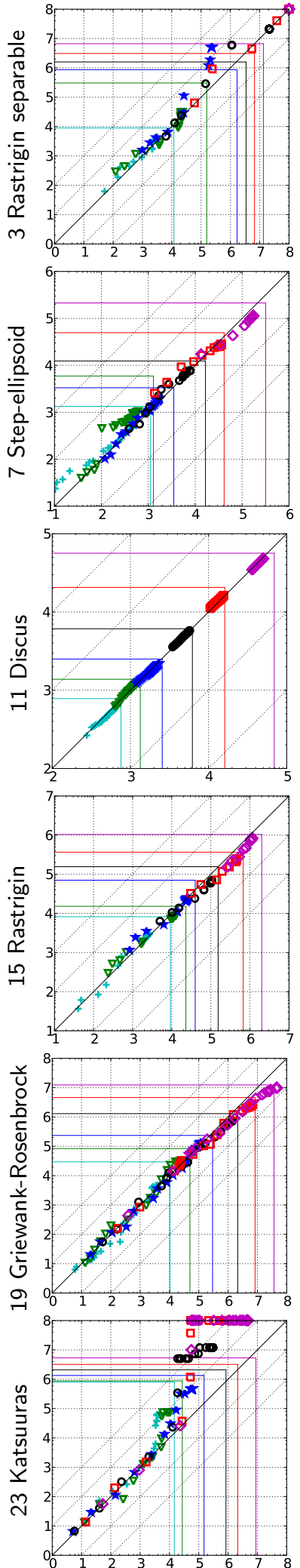
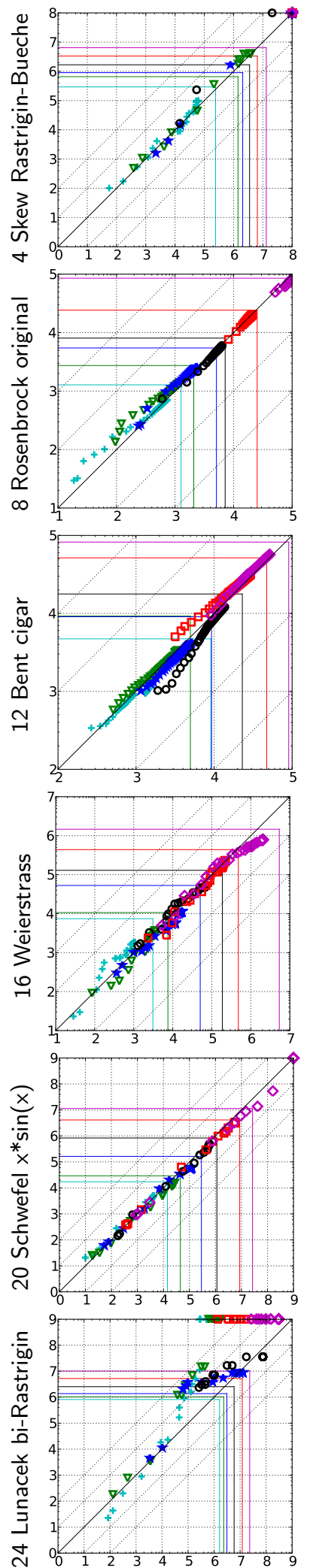

Figure 2: Expected running time (ERT in log10 of number of function evaluations) of IPOP-CMA versus BIPOP-CMA for 46 target values $\Delta f \in\left[10^{-8}, 10\right]$ in each dimension for functions $f_{1}-f_{24}$. Markers on the upper or right egde indicate that the target value was never reached by IPOP-CMA or BIPOP-CMA respectively. Markers represent dimension: $2:+, 3: \nabla, 5: \star, 10: \circ, 20: \square, 40: \diamond$. 

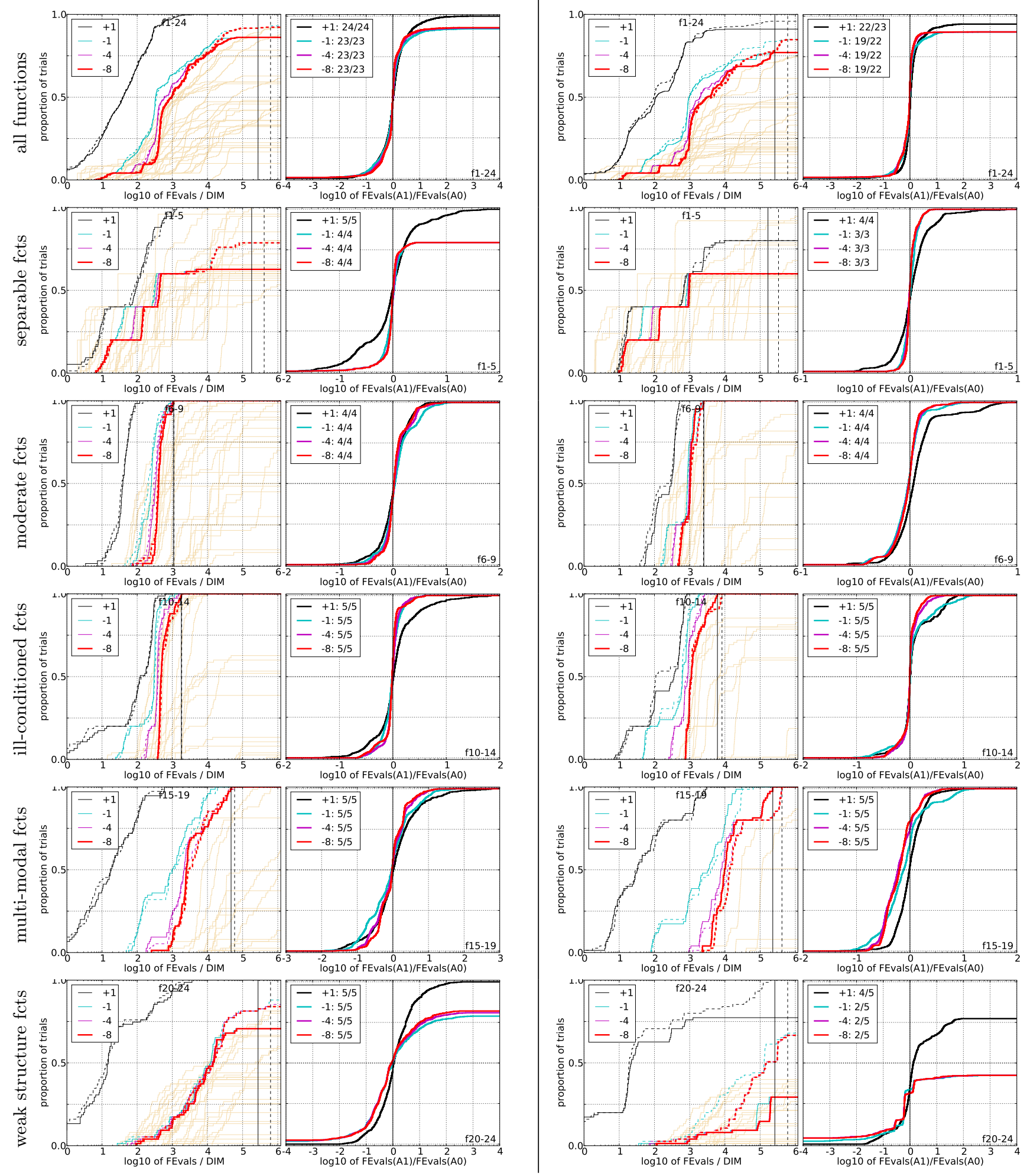

Figure 3: Empirical cumulative distributions (ECDF) of run lengths and speed-up ratios in 5-D (left) and 20D (right). Left sub-columns: ECDF of the number of function evaluations divided by dimension $D$ (FEvals/D) to reach a target value $f_{\mathrm{opt}}+\Delta f$ with $\Delta f=10^{k}$, where $k \in\{1,-1,-4,-8\}$ is given by the first value in the legend, for IPOP-CMA (solid) and BIPOP-CMA (dashed). Light beige lines show the ECDF of FEvals for target value $\Delta f=10^{-8}$ of algorithms benchmarked during BBOB-2009. Right sub-columns: ECDF of FEval ratios of IPOP-CMA divided by BIPOP-CMA, all trial pairs for each function. Pairs where both trials failed are disregarded, pairs where one trial failed are visible in the limits being $>0$ or $<1$. The legends indicate the number of functions that were solved in at least one trial (IPOP-CMA first). 
$5-\mathrm{D}$

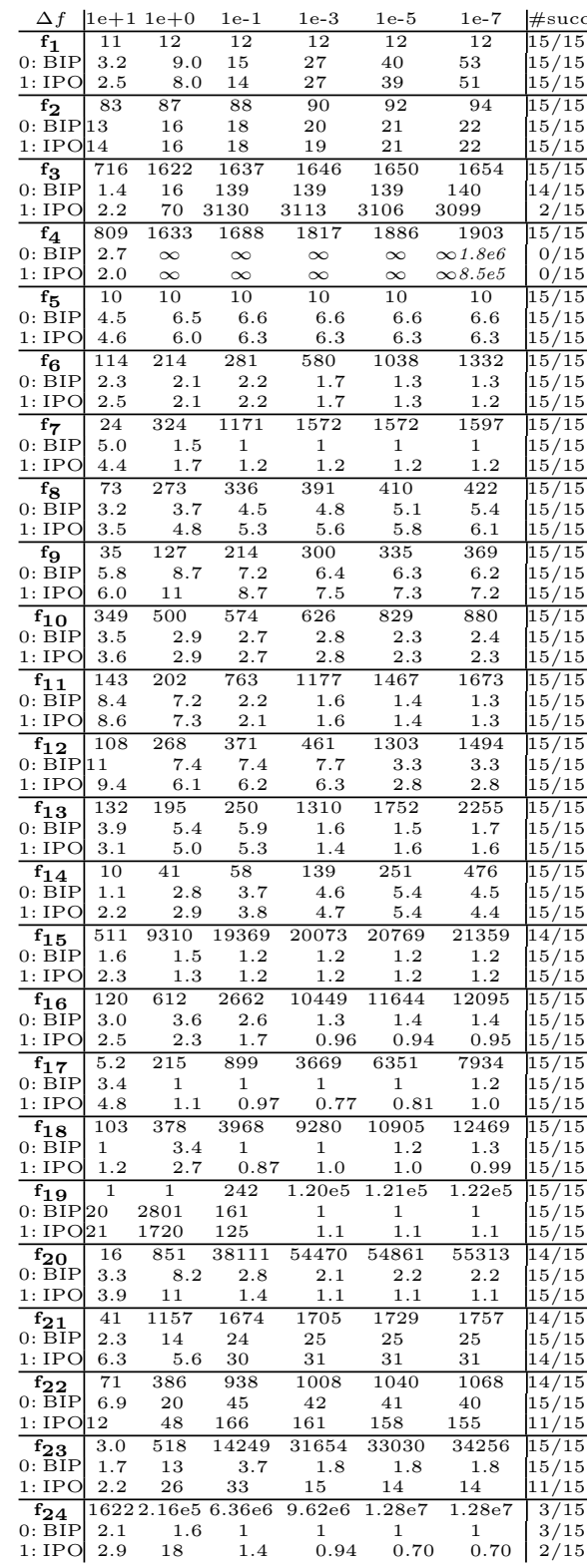

$20-\mathrm{D}$

\begin{tabular}{|c|c|c|c|c|c|c|c|}
\hline$\Delta f$ & $1 \mathrm{e}+1$ & $1 \mathrm{e}+0$ & $1 \mathrm{e}-1$ & $1 \mathrm{e}-3$ & $1 e-5$ & $1 e-7$ & \#succ \\
\hline$f_{1}$ & 43 & 43 & 43 & 43 & 43 & 43 & $15 / 15$ \\
\hline 0: BIP & 7.9 & 14 & 20 & 33 & 45 & 57 & $15 / 15$ \\
\hline 1: IPO & 8.0 & 14 & 20 & 33 & 46 & 58 & $15 / 15$ \\
\hline \begin{tabular}{l|}
$f_{2}$ \\
\end{tabular} & 385 & 386 & 387 & 390 & 391 & 393 & $15 / 15$ \\
\hline $0:$ BIP & 35 & 40 & 44 & 47 & 48 & 50 & $15 / 15$ \\
\hline 1: IPO & 35 & 41 & 43 & 45 & 47 & 48 & $15 / 15$ \\
\hline \begin{tabular}{l|}
$f_{3}$ \\
\end{tabular} & 5066 & 7626 & 7635 & 7643 & 7646 & 7651 & $15 / 15$ \\
\hline $0:$ BIP & 12 & $\infty$ & $\infty$ & $\infty$ & $\infty$ & $\infty 5.7 e 6$ & $0 / 15$ \\
\hline 1: IPO & 13 & $\infty$ & $\infty$ & $\infty$ & $\infty$ & $\infty 2.9 e 6$ & $0 / 15$ \\
\hline $\mathrm{f}_{4}$ & 4722 & 7628 & 7666 & 7700 & 7758 & $1.41 \mathrm{e} 5$ & $9 / 15$ \\
\hline $0:$ BIP & $\infty$ & $\infty$ & $\infty$ & $\infty$ & $\infty$ & $\infty 5.5 e 6$ & $0 / 15$ \\
\hline 1: IPO & $\infty$ & $\infty$ & $\infty$ & $\infty$ & $\infty$ & $\infty 2.8 \mathrm{e} 6$ & $0 / 15$ \\
\hline$f_{5}$ & 41 & 41 & 41 & 41 & 41 & 41 & $15 / 15$ \\
\hline $0: \mathrm{BIP}$ & 5.1 & 6.2 & 6.3 & 6.3 & 6.3 & 6.3 & $15 / 15$ \\
\hline 1: IPO & 5.8 & 6.5 & 6.7 & 6.7 & 6.7 & 6.7 & $15 / 15$ \\
\hline $\mathrm{f}_{6}$ & 1296 & 2343 & 3413 & 5220 & 6728 & 8409 & $15 / 15$ \\
\hline $0: \mathrm{BIP}$ & 1.5 & 1.3 & 1.2 & 1.1 & 1.2 & 1.2 & $15 / 15$ \\
\hline 1: IPO & \begin{tabular}{|l}
1.7 \\
\end{tabular} & 1.3 & 1.2 & 1.2 & 1.2 & 1.2 & $15 / 15$ \\
\hline$f_{7}$ & 1351 & 4274 & 9503 & 16524 & 16524 & 16969 & $15 / 15$ \\
\hline 0: BIP & 1 & 4.9 & 3.5 & 2.2 & 2.2 & 2.1 & $15 / 15$ \\
\hline 1: IPO & 1.9 & 4.8 & 2.7 & $1.7^{\star}$ & $1.7^{\star}$ & $1.6^{\star}$ & $15 / 15$ \\
\hline$f_{8}$ & 2039 & 3871 & 4040 & 4219 & 4371 & 4484 & $15 / 15$ \\
\hline 0: BIP & 4.0 & 4.0 & 4.3 & 4.5 & 4.6 & 4.6 & $15 / 15$ \\
\hline 1: IPO & 3.7 & 3.9 & 4.2 & 4.4 & 4.4 & 4.5 & $15 / 15$ \\
\hline \begin{tabular}{l|}
$f_{9}$ \\
\end{tabular} & 1716 & 3102 & 3277 & 3455 & 3594 & 3727 & $15 / 15$ \\
\hline $0: \mathrm{BIP}$ & 4.7 & 5.7 & 6.0 & 6.1 & 6.1 & 6.1 & $15 / 15$ \\
\hline 1: IPO & 4.6 & 5.7 & 6.0 & 6.1 & 6.1 & 6.1 & $15 / 15$ \\
\hline$f_{10}$ & 7413 & 8661 & 10735 & 14920 & 17073 & 17476 & $15 / 15$ \\
\hline $0:$ BIP & 1.9 & 1.8 & 1.6 & 1.2 & 1.1 & 1.1 & $15 / 15$ \\
\hline $1:$ IPO & 1.8 & 1.8 & 1.5 & 1.2 & 1.1 & 1.1 & $15 / 15$ \\
\hline$f_{11}$ & 1002 & 2228 & 6278 & 9762 & 12285 & 14831 & $15 / 15$ \\
\hline $0: \mathrm{BIP}$ & 10 & 5.1 & 1.9 & 1.4 & 1.2 & 1.0 & $15 / 15$ \\
\hline 1: IPO & 11 & 5.4 & 2.1 & 1.4 & 1.2 & 1.1 & $15 / 15$ \\
\hline $\begin{array}{l}f_{12} \\
\end{array}$ & 1042 & 1938 & 2740 & 4140 & 12407 & 13827 & $15 / 15$ \\
\hline $0: \mathrm{BIP}$ & 3.0 & 4.0 & 4.5 & 4.5 & 1.9 & 2.0 & $15 / 15$ \\
\hline 1: IPO & 4.8 & 5.3 & 5.5 & 5.1 & 2.1 & 2.2 & $15 / 15$ \\
\hline \begin{tabular}{l|}
$f_{13}$ \\
\end{tabular} & 652 & 2021 & 2751 & 18749 & 24455 & 30201 & $15 / 15$ \\
\hline $0:$ BIP & 4.3 & 2.7 & 5.1 & 1.5 & 2.3 & 3.0 & $15 / 15$ \\
\hline 1: IPO & 6.5 & 4.8 & 6.2 & 1.4 & 1.7 & 2.3 & $15 / 15$ \\
\hline$f_{14}$ & 75 & 239 & 304 & 932 & 1648 & 15661 & $15 / 15$ \\
\hline $0:$ BIP & 3.9 & 2.9 & 3.7 & 4.1 & 6.2 & 1.2 & $15 / 15$ \\
\hline 1: IPO & 3.7 & 2.8 & 3.6 & 3.9 & 6.0 & 1.2 & $15 / 15$ \\
\hline \begin{tabular}{l|}
$f_{15}$ \\
\end{tabular} & 30378 & $1.47 \mathrm{e} 5$ & $3.12 \mathrm{e} 5$ & $3.20 \mathrm{e} 5$ & $4.49 \mathrm{e} 5$ & $4.59 \mathrm{e} 5$ & $15 / 15$ \\
\hline $0:$ BIP & 1 & 2.0 & 1.4 & 1.4 & 1 & 1 & $15 / 15$ \\
\hline $1:$ IPO & 1.1 & $1.1^{\star}$ & $0.69^{\star}$ & $0.70^{\star}$ & $0.52^{\star \downarrow}$ & $0.53^{\star \downarrow}$ & $15 / 15$ \\
\hline$f_{16}$ & 1384 & 27265 & 77015 & $1.88 \mathrm{e} 5$ & $1.98 \mathrm{e} 5$ & $2.20 \mathrm{e} 5$ & $15 / 15$ \\
\hline $0: \mathrm{BIP}$ & 1.7 & 1.0 & 1.2 & 1 & 1 & 1 & $15 / 15$ \\
\hline 1: IPO & 1.7 & 0.81 & 0.92 & 0.84 & 1.1 & 1.0 & $15 / 15$ \\
\hline \begin{tabular}{l|}
$f_{17}$ \\
\end{tabular} & 63 & 1030 & 4005 & 30677 & 56288 & 80472 & $15 / 15$ \\
\hline $0: \mathrm{BIP}$ & 2.2 & 1 & 1 & 1.2 & 1.3 & 1.4 & $15 / 15$ \\
\hline 1: IPO & 2.1 & 0.94 & 1.2 & 0.76 & 0.99 & 1.0 & $15 / 15$ \\
\hline \begin{tabular}{l|}
$f_{18}$ \\
\end{tabular} & 621 & 3972 & 19561 & 67569 & $1.31 \mathrm{e} 5$ & $1.47 \mathrm{e} 5$ & $15 / 15$ \\
\hline $0:$ BIP & 1.0 & 2.4 & 1.2 & 1.1 & 1.7 & 1.6 & $15 / 15$ \\
\hline $1:$ IPO & 1.1 & 1.8 & 1.1 & 0.97 & $1.0^{\star}$ & $1.1 \star 2$ & $15 / 15$ \\
\hline$f_{19}$ & 1 & 1 & $3.43 \mathrm{e} 5$ & $6.22 \mathrm{e} 6$ & $6.69 \mathrm{e} 6$ & $6.74 \mathrm{e} 6$ & $15 / 15$ \\
\hline $0: \mathrm{BIP}$ & 169 & 23770 & 1.2 & 1 & 1 & 1 & $15 / 15$ \\
\hline 1: IPO & 161 & 27333 & 0.71 & $0.38^{\star 3 \downarrow}$ & $0.41 \star 3 \downarrow 3$ & $0.41 \star 3 \downarrow 3$ & $315 / 15$ \\
\hline \begin{tabular}{l|}
$f_{20}$ \\
\end{tabular} & 82 & 46150 & $3.10 \mathrm{e} 6$ & $5.54 \mathrm{e} 6$ & $5.59 \mathrm{e} 6$ & $5.64 \mathrm{e} 6$ & $14 / 15$ \\
\hline $0: \mathrm{BIP}$ & 4.3 & 9.2 & 1 & 1 & 1 & 1 & $14 / 15$ \\
\hline 1: IPO & 4.6 & 6.4 & 0.65 & 0.57 & 0.58 & 0.58 & $15 / 15$ \\
\hline $\mathbf{f}_{21}$ & 561 & 6541 & 14103 & 14643 & 15567 & 17589 & $15 / 15$ \\
\hline $0: \mathrm{BIP}$ & 3.2 & 55 & 48 & 46 & 43 & 39 & $13 / 15$ \\
\hline 1: IPO & 3.7 & 139 & 110 & 106 & 100 & 88 & $7 / 15$ \\
\hline$f_{22}$ & 467 & 5580 & 23491 & 24948 & 26847 & $1.35 \mathrm{e} 5$ & $12 / 15$ \\
\hline $0:$ BIP & 6.8 & 13 & $215^{\star}$ & $202^{\star}$ & $188^{\star}$ & $37^{\star}$ & $5 / 15$ \\
\hline 1: IPO & 445 & 287 & $\infty$ & $\infty$ & $\infty$ & $\infty 1.3 \mathrm{e} 6$ & $0 / 15$ \\
\hline $\begin{array}{l}f_{23} \\
\end{array}$ & 3.2 & 1614 & 67457 & $4.89 \mathrm{e} 5$ & $8.11 \mathrm{e} 5$ & $8.38 \mathrm{e} 5$ & $15 / 15$ \\
\hline $0: \mathrm{BIP}$ & 4.3 & $32^{\star 3}$ & $1 \star 3$ & $2.0^{\star 3}$ & $1.2^{\star 3}$ & $1.2^{\star 3}$ & $15 / 15$ \\
\hline $1: \mathrm{IPO}$ & 4.3 & 23082 & $\infty$ & $\infty$ & $\infty$ & $\infty 2.5 e 6$ & $0 / 15$ \\
\hline \begin{tabular}{l|}
$f_{24}$ \\
\end{tabular} & $1.34 \mathrm{e} 6$ & $7.48 \mathrm{e} 6$ & $5.19 \mathrm{e} 7$ & $5.20 \mathrm{e} 7$ & $5.20 \mathrm{e} 7$ & $5.20 \mathrm{e} 7$ & $3 / 15$ \\
\hline 0 : BIP & $1 \star 3$ & $1 \star 3$ & $1 \star 3$ & $1 \star 3$ & $1 \star 3$ & $1 \star 3$ & $3 / 15$ \\
\hline 1: IPO & $\infty$ & $\infty$ & $\infty$ & $\infty$ & $\infty$ & $\infty 5.1 \mathrm{e} 6$ & $0 / 15$ \\
\hline
\end{tabular}

Table 1: Expected running time (ERT in number of function evaluations) divided by the best ERT measured during BBOB-2009 (given in the respective first row) for different $\Delta f$ values for functions $f_{1}-f_{24}$. The median number of conducted function evaluations is additionally given in italics, if ERT $\left(10^{-7}\right)=\infty$. \#succ is the number of trials that reached the final target $f_{\text {opt }}+10^{-8}$. 0: BIP is BIPOP-CMA and 1: IPO is IPOP-CMA. Bold entries are statistically significantly better compared to the other algorithm, with $p=0.05$ or $p=10^{-k}$ where $k>1$ is the number following the $\star$ symbol, with Bonferroni correction of 48 . 


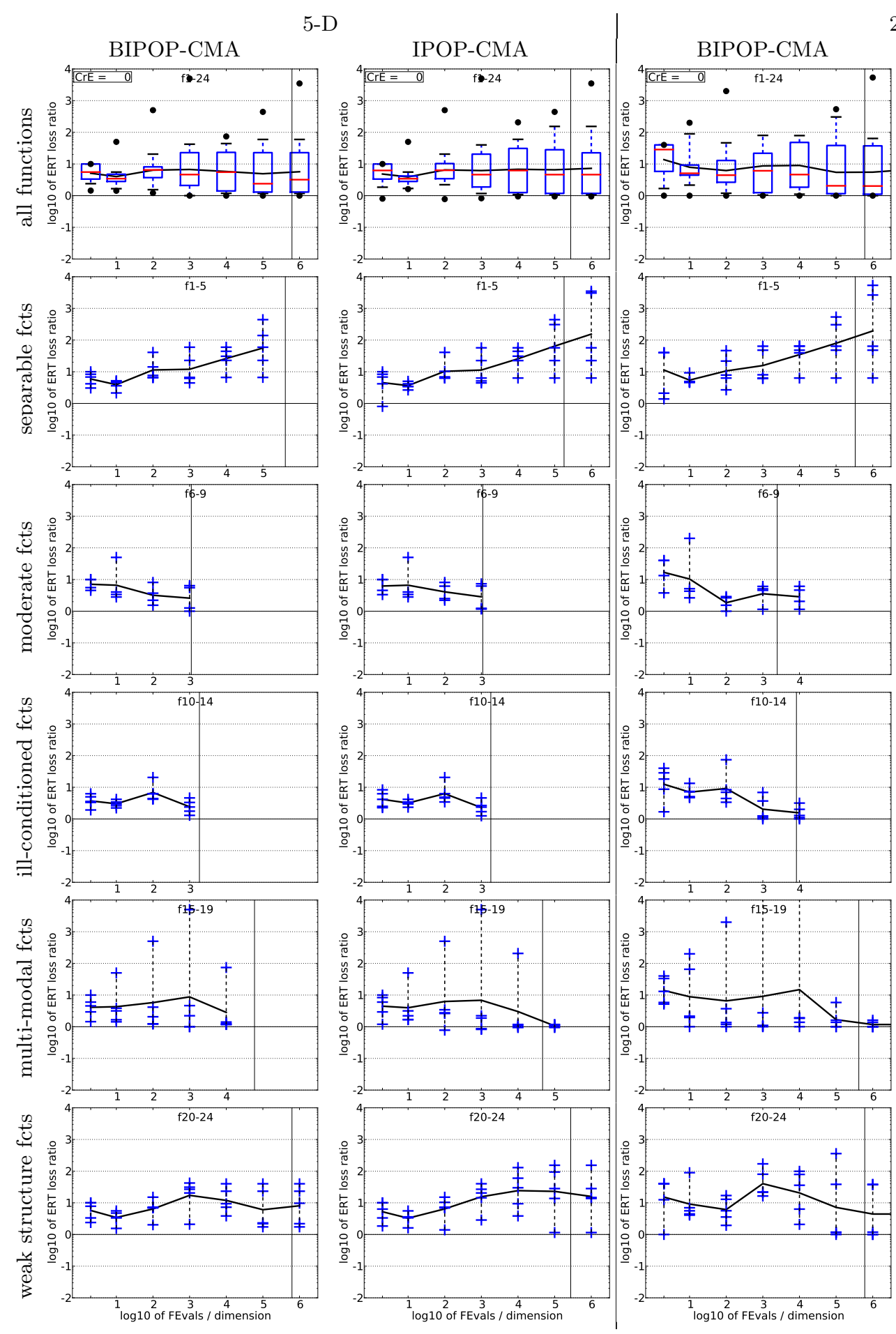

$20-\mathrm{D}$

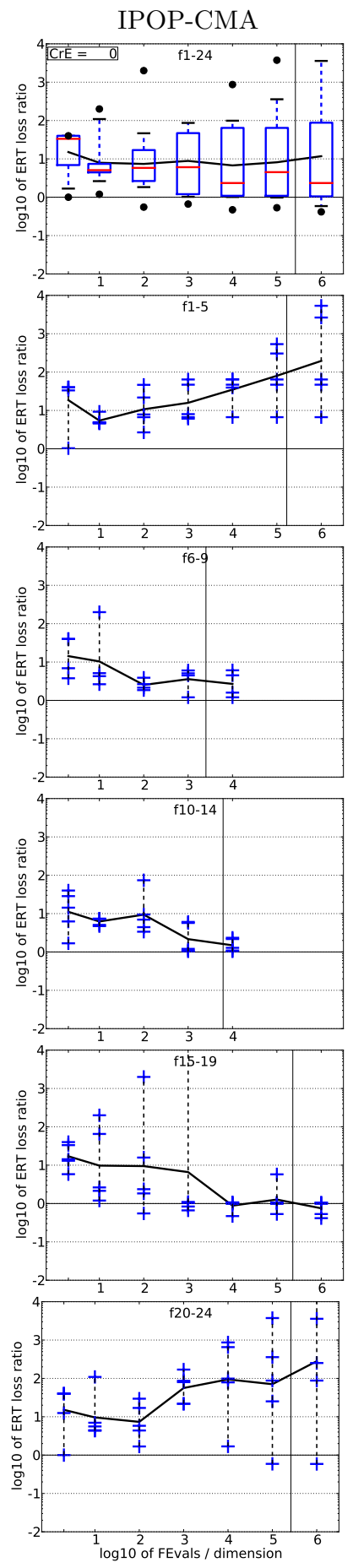

Figure 4: ERT loss ratio versus given budget FEvals. The target value $f_{\mathrm{t}}$ for ERT is the smallest (best) recorded function value such that $\operatorname{ERT}\left(f_{\mathrm{t}}\right) \leq$ FEvals for the presented algorithm. Shown is FEvals divided by the respective best $\operatorname{ERT}\left(f_{\mathrm{t}}\right)$ from BBOB-2009 for functions $f_{1}-f_{24}$ in 5-D and 20-D. Each ERT is multiplied by $\exp (\mathrm{CrE})$ correcting for the parameter crafting effort. Line: geometric mean. Box-Whisker error bar: 25-75\%-ile with median (box), 10-90\%-ile (caps), and minimum and maximum ERT loss ratio (points). The vertical line gives the maximal number of function evaluations in this function subset. 
Table 2: ERT loss ratio (see Figure 4) compared to the respective best result from BBOB-2009 for budgets given in the first column. The last row $R L_{U S} / D$ gives the number of function evaluations in unsuccessful runs divided by dimension. Shown are the smallest, $10 \%$-ile, $25 \%$-ile, $50 \%$-ile, $75 \%$-ile and $90 \%$-ile value (smaller values are better). ERT Loss ratio is equal to zero if the algorithm considered outperformed all algorithms from BBOB-2009.

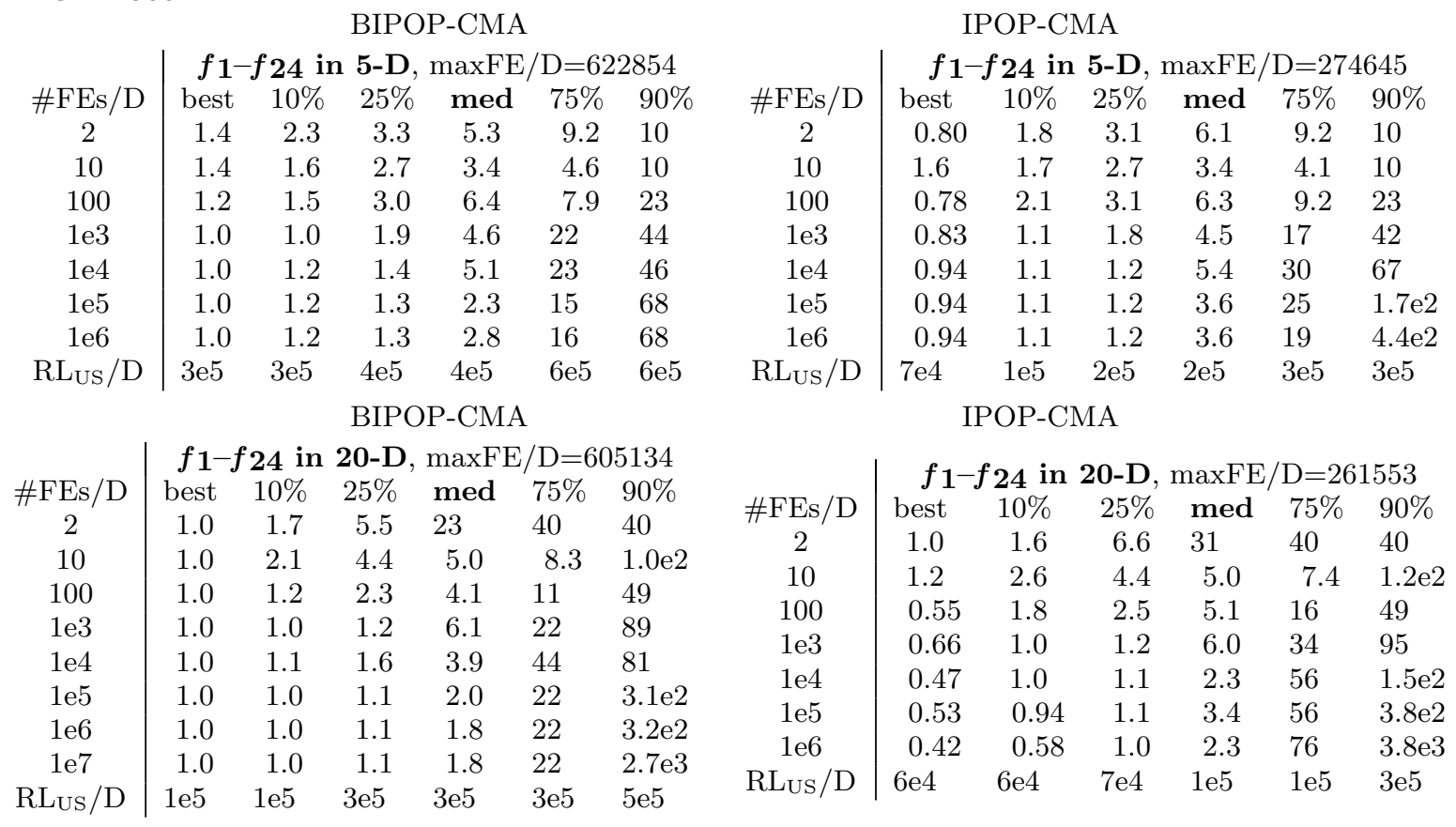

